\title{
GENETIC ALGORITHM-BASED DYNAMIC INTRAOPERATIVE TREATMENT PLANNING FOR PROSTATE BRACHYTHERAPY
}

\author{
Lei Fu, Yan Yu and Haisong Liu \\ Departments of Radiation Oncology, University of Rochester, Rochester, NY, U.S.A
}

\begin{abstract}
This paper reports on the use of a genetic algorithm (GA) for dynamic intraoperative treatment planning (DITP) with stepwise replanning for the prostate implant. The study applied a simple needle and seed reconstruction model to simulate the seed displacement. The simulation of DITP consists of an initial plan and four re-optimized plans. The results show that, on average, the total number of seeds required by each re-plan increased. The average maximum urethral dose and maximum rectal dose showed a slight increase during the first two replanning steps, but increased substantially during the latter two replanning steps. Several issues are discussed regarding the limitation and future improvement of GA-based DITP.
\end{abstract}

Key words: Prostate, Brachytherapy, Treatment planning, Intraoperative, Genetic algorithm, Robotics, Dosimetry

\section{INTRODUCTION}

Prostate brachytherapy is a method of treating early prostate cancer using permanent implantation of radioactive sources. Traditionally, the implantation pattern was preplanned days or weeks before surgery. In recent years, intraoperative preplanning and interactive planning are gaining popularity because of their advantages over the preplanned technique. Furthermore, a number of institutions are currently performing and refining dynamic intraoperative treatment planning (DITP), which was defined by the American Brachytherapy Society as continuous update of the treatment plan in the operating room (OR) [1]. Potentially, DITP can address and 
compensate for defects in the dosimetry due to deflection of needles and displacement of seed positions, and due to changes in prostate size and shape. The major limitation of DITP is the difficulty in localizing the seeds in relation to the prostate in real time. Memorial Sloan-Kettering Cancer Center used the technique of fusion of ultrasound and fluoroscopy to achieve seed reconstruction $[2,3]$. They recently presented an intraoperative dynamic dose optimization (IDDO) algorithm that can theoretically perform the DITP. IDDO re-optimizes the plan 2, 3, or 4 times during the implant based on the simulated shifted seed positions. They analyzed the sensitivity and effect of the IDDO procedure with respect to the total number of re-optimizations performed, and reported that IDDO treatment planning can improve postimplant dose coverage and conformity and reduced irradiation to urethra and rectum [2].

In our center, a robotic system for assisting prostate brachytherapy is under development. It will provide the possibility of DITP because of improvements in active, on-demand image acquisition and seeds detection. Our inverse planning system applies needle and seed spacing optimizations using a genetic algorithm (GA), which is different from the integer programming technique used in MSKCC's 3D treatment plan system.

In this paper, a simulation of DITP is presented using a model of seed displacement based on clinical observation in our center. This simulation consists of an initial plan and four re-optimized plans. Each re-optimized plan is based on the previous simulated position of seeds. Our study focuses on the dosimetric analysis and comparison of each re-optimization step.

\section{METHODS AND MATERIALS}

\subsection{Simulation of seeds displacement}

The implementation of DITP requires dosimetric information based on the actual positions of seeds already implanted. Some center applied Monte Carlo models to simulate post-implant needle reconstruction [4]. The seed displacement statistics varies among centers because of different implantation techniques.

According to our clinical observation, we created a simple but effective model to simulate the displacement of implanted seeds. In this model, postimplant seed displacements were decomposed into needle displacements and 
seed displacements relative to the needle. If $x_{p i}, y_{p i}, z_{p i}$ are the coordinates of the $i$ th seed of a n-source planning needle (there are total $m$ needles to be displaced), and $x_{q i}, y_{q i}, z_{q i}$ are the coordinates of simulated post-implant source, the transformations from planning to post-implant are applied as follows:

1. Needle translation $\delta x j, \delta y j$.

$$
\begin{aligned}
& x_{\mathrm{qi}}=\mathrm{x}_{\mathrm{pi}}+\delta \mathrm{x}_{\mathrm{j}}, \\
& \mathrm{yqi}=\mathrm{ypi}+\delta y \mathrm{j},
\end{aligned}
$$$$
i=1, \ldots, n, j=1, \ldots, m
$$

2. Angulation. The angles $\alpha j$ and $\beta_{j}$ respectively formed by the needle and the insertion axis $z$ in the coronal and sagittal planes.

$$
\begin{aligned}
& \mathrm{z}_{\mathrm{qi}}=\mathrm{z}_{\mathrm{pi}} /\left(1+\tan \left(\alpha_{\mathrm{j}}\right)^{2}+\tan \left(\beta_{\mathrm{j}}\right)^{2}\right)^{1 / 2}, \\
& \mathrm{x}_{\mathrm{qi}}=\mathrm{z}_{\mathrm{pi}} \cdot \tan \left(\alpha_{\mathrm{j}}\right)+\mathrm{x}_{\mathrm{pi}}, \\
& \mathrm{yqi}=\mathrm{zpi} \cdot \tan (\beta \mathrm{j})+\mathrm{ypi},
\end{aligned}
$$$$
i=1, \ldots, n, j=1, \ldots, m
$$

3. Random noise of seeds relative to the needle: $\Delta x_{i}, \Delta y_{i}, \Delta z_{i}$

$$
\begin{aligned}
& x_{q i}=x_{q i}+\Delta x_{i}, \\
& y_{q i}=y_{q i}+\Delta y_{i}, \\
& z_{q i}=z_{q i}+\Delta z_{i},
\end{aligned}
$$$$
i=1, \ldots, n \text {, }
$$

The actual seed positions were identified from CT images, which were obtained one day after the implant procedure. The actual seed positions were automatically matched with the original planed seed positions using a deterministic annealing point-match algorithm. The mean and standard deviation of parameters $\delta x_{j}, \delta y_{j}, \alpha j, \beta_{j}, \Delta x_{i}, \Delta y_{i}$, and $\Delta z_{i}$ were collected from 15 patients. They were, respectively, $0 \pm 0.13 \mathrm{~cm}, 0 \pm 0.14 \mathrm{~cm}, 0 \pm 4.4^{\circ}$, 
$0.08^{\circ} \pm 5.5^{\circ}, 0 \pm 0.04 \mathrm{~cm}, 0 \pm 0.05 \mathrm{~cm}$, and $0 \pm 0.26 \mathrm{~cm}$. We used this model to obtain simulated individual seed displacement to approximate practical cases. The mean and standard deviation in $\mathrm{x}, \mathrm{y}, \mathrm{z}$ of individual seed displacement are $0.15 \mathrm{~cm}, 0.17 \mathrm{~cm}, 0.26 \mathrm{~cm}$, respectively.

\subsection{Genetic algorithm optimization}

Our treatment planning software employs GA as the optimization engine because of its conceptual simplicity, the ease of programming, and the small number of parameters to be defined. In this algorithm, grid coordinates $\mathrm{x}$ and $y$, angles $\alpha$ and $\beta$ represent the solid angle position of each needle, and each needle position can be set to 1 or 0 , which indicates if this position will be selected in the plan. This is an improvement based on our previous GA software, where only the grid coordinates $\mathrm{x}$ and $\mathrm{y}$ represent each needle position. Therefore, current software can generate traditional parallel needle dosimetry, as well as conical dosimetry and hybrid dosimetry where both parallel needles and angulated needles are applied.

There are two steps of optimization in the GA software: the needle optimization and seed spacing optimization. First, the needle optimization was performed by using one of a few selected seed spacing rules $[5,6]$. Nominally, seeds were $1 \mathrm{~cm}$ apart center-to-center in the same needle, and $0.5 \mathrm{~cm}$ spacing was permitted at the apex or base to best fit the length of the needle path in the prostate. This restriction in the original algorithm was introduced to permit use of ready-loaded needles or stranded seeds, which were typically provided in this fashion. All needles that intersected the prostate boundary become the chromosomes. During the GA optimization, the selected seed loading rule was applied to calculate the seed coordinates, and then the seed positions were used to calculate the dose, from which the fitness function was evaluated. The fitness function is essentially a weighted summation of the dosimetric outcomes. After a certain number of iterations (200 generations were used in our study), an optimal needle pattern was obtained.

Second, seed spacing optimization is performed based on this needle pattern [7]. Assuming $0.5 \mathrm{~cm}$ minimum nominal spacing and increment of seeds within each needle, all possible seed positions within the optimal needle pattern constitute the GA chromosomes. Using the same fitness function, an optimal seed position can be obtained. 


\subsection{Simulation of intraoperative treatment planning}

The simulated DITP consists of one initial optimized plan and four intraoperative optimized plans.

First, the GA-based inverse planning software was used to obtain an initial optimized dosimetry. Based on this result, about $1 / 4$ of the total number of needles were selected for "simulated implantation". The seeds in these needles were displaced using the model described above in this paper. The needles were selected in the following order: right periphery of prostate, then left periphery of prostate, and lastly posterior to anterior. The left or right periphery is defined as the left or right $1 / 4$ width of the prostate in the transverse view.

Second, the remaining volume of the prostate was re-optimized using the same inverse planning algorithm. In this step the positions of the seeds already deposited in the prostate were included in the dosimetry calculation. The possible remaining positions were those that were certain distance away from the implanted seeds. This distance is a variable parameter in our software, and we used $2.5 \mathrm{~mm}$ in this study according to the parameter test results, which will be discussed later in this paper.

Third, the above method of seed displacement and re-optimization was repeated twice. Each time $1 / 3$ and $1 / 2$, respectively, of the remaining needles and seeds were displaced. The positions of the seeds that were already displaced in the previous step were kept unchanged.

Lastly, all the remaining needles and seeds were displaced and the implant was re-optimized to see if any additional seed need to be placed.

\section{$2.4 \quad$ Test cases}

Ten unselected prostate seed implant cases were used in this study. All necessary data were acquired in a previous study [8]. The prostate volume ranges from 15 to $66 \mathrm{~cm}^{3}$. For each case, two types of seeds and different prescription doses were tested: iodine-125 with initial air kerma strength of $0.5 \mathrm{U}$ and a prescription dose of $145 \mathrm{~Gy}$, and palladium-103 with initial air kerma strength of $2.5 \mathrm{U}$ and a prescription dose of $115 \mathrm{~Gy}$. Only one case, which is the smallest prostate, used $2.2 \mathrm{U}$ per seed. 
Each simulation was repeated three times to obtain the average results, because the random displacement of seeds tends to influence the final dosimetric results.

The following data were recorded for dosimetric analysis: the number of seeds used, maximum urethral dose (MUD), and maximum rectal dose (MRD). The change of the number of seeds is computed as the ratio of the total number of seeds used in each step (including those seeds already implanted) to the number of seeds used in the initial plan. We are interested in the change in the number of seeds, which indicates the change of S10mPD (total air kerma strength to deliver 10 Gy minimum peripheral dose). Any increase of S10mPD also indicates worsening of dose uniformity.

\section{RESULTS}

Table 1 shows the change in the number of seeds comparing the initial plan with each re-optimized plan. In general, the average number of seeds increased during the DITP simulation. The variation in the percentage change of seeds needed is also substantial. (The number in the parentheses in the tables shows the variation from minimum to maximum across ten cases.)

Table 1. Change of number of seeds, comparing each re-optimization with initial plan

\begin{tabular}{llllll}
\hline $\begin{array}{l}\text { Seed } \\
\text { type }\end{array}$ & $\begin{array}{l}\text { Percentage } \\
\text { change of } \\
\text { number of } \\
\text { seeds }\end{array}$ & $\begin{array}{l}\text { Re } \\
\text { optimization 1 }\end{array}$ & $\begin{array}{l}\text { Re } \\
\text { optimization 2 }\end{array}$ & $\begin{array}{l}\text { Re- } \\
\text { optimization 3 }\end{array}$ & $\begin{array}{l}\text { Re- } \\
\text { optimization 4 }\end{array}$ \\
\hline I-125 & Mean & $-1(--7 \sim 5)$ & $1(-8 \sim 15)$ & $5(-5 \sim 23)$ & $11(0 \sim 37)$ \\
& Standard Dev. & 4 & 7 & 8 & 11 \\
Pd-103 & Mean & $3(-2 \sim 7)$ & $6(-2 \sim 18)$ & $11(3 \sim 25)$ & $21(7 \sim 35)$ \\
& Standard Dev. & 3 & 5 & 7 & 9 \\
\hline
\end{tabular}

Figures 1 and 2 illustrate the percentage change of number of seeds in each re-optimization step for the 10 cases, using seed type I-125 and Pd-103 respectively. Both results show the same trend of considerable variation among cases. However, Pd-103 produces more change of seeds than I-125. 


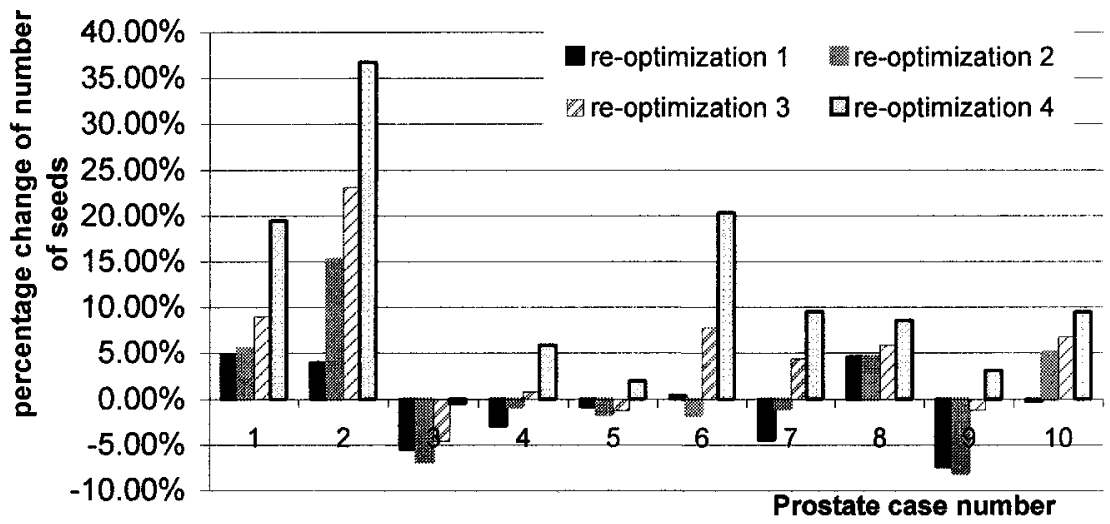

Figure 1. Change number of seeds in each re-optimization for 10 cases; using I-125

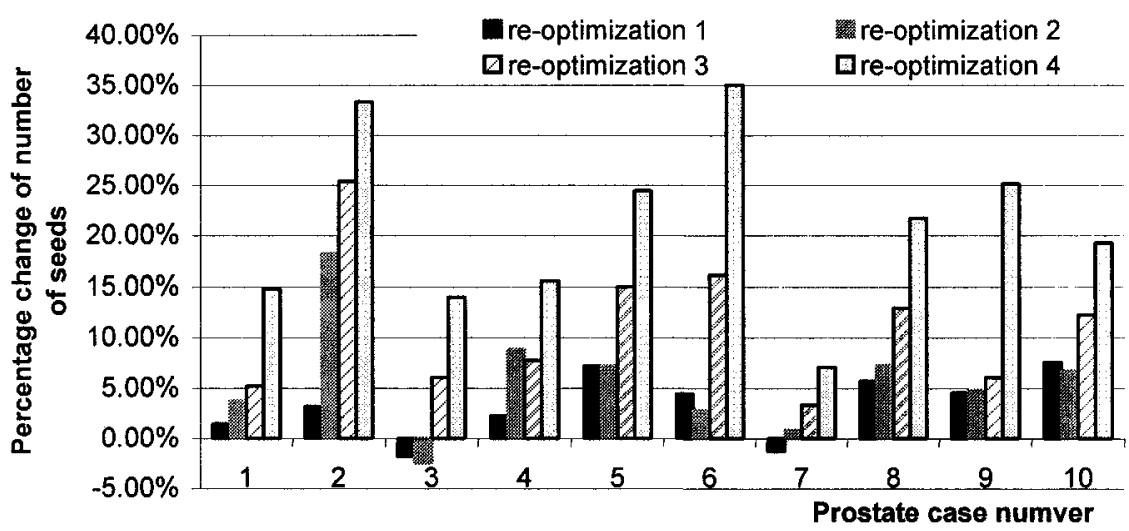

Figure 2. Change number of seeds in each re-optimization for 10 cases; using Pd-103

Table 2 and Table 3, respectively, show the change of MUD and MRD comparing the initial plan with each re-optimized plan. The average MUD and MRD also increased during re-optimization, particularly in the third reoptimization. Recall that during the third optimization, about $3 / 4$ of the total seeds were displaced. These seeds include some located in the center of the prostate, which have relatively large influence to the MUD and MRD. At the last optimization, all of the seeds were displaced and the uniformity of prostate dosimetry became worst. The results also suggest that iodine and 
palladium have similar effects on the change of MUD and MRD, although I125 displayed somewhat better test results than Pd-103.

Table 2. Change of MUD, comparing each re-optimization with initial plan

\begin{tabular}{llllll}
\hline $\begin{array}{l}\text { Seed } \\
\text { type }\end{array}$ & $\begin{array}{l}\text { Percentage } \\
\text { change of } \\
\text { number of } \\
\text { seeds }\end{array}$ & $\begin{array}{l}\text { Re- } \\
\text { optimization 1 }\end{array}$ & $\begin{array}{l}\text { Re- } \\
\text { optimization 2 }\end{array}$ & $\begin{array}{l}\text { Re- } \\
\text { optimization 3 }\end{array}$ & $\begin{array}{l}\text { Re- } \\
\text { optimization 4 }\end{array}$ \\
\hline I-125 & Mean & $-4(-19 \sim 11)$ & $-4(-20 \sim 25)$ & $3(-9 \sim 73)$ & $17(-9 \sim 73)$ \\
& Standard Dev. & 9 & 12 & 20 & 29 \\
Pd-103 & Mean & $1(-8 \sim 8)$ & $-1(-12 \sim 13)$ & $9(-15 \sim 25)$ & $28(7 \sim 60)$ \\
& Standard Dev. & 6 & 8 & 10 & 14 \\
\hline
\end{tabular}

Table 3. Change of MRD, comparing each re-optimization with initial plan

\begin{tabular}{llllll}
\hline $\begin{array}{l}\text { Seed } \\
\text { type }\end{array}$ & $\begin{array}{l}\text { Percentage } \\
\text { change of } \\
\text { number of } \\
\text { seeds }\end{array}$ & $\begin{array}{l}\text { Re- } \\
\text { optimization 1 }\end{array}$ & $\begin{array}{l}\text { Re- } \\
\text { optimization 2 }\end{array}$ & $\begin{array}{l}\text { Re- } \\
\text { optimization 3 }\end{array}$ & $\begin{array}{l}\text { Re- } \\
\text { optimization 4 }\end{array}$ \\
\hline I-125 & Mean & $0(-13 \sim 17)$ & $2(-14 \sim 29)$ & $10(-8 \sim 38)$ & $16(-7 \sim 52)$ \\
& Standard Dev. & 10 & 12 & 13 & 17 \\
Pd-103 & Mean & $8(-5 \sim 24)$ & $21(0 \sim 66)$ & $35(0 \sim 94)$ & $42(-1 \sim 99)$ \\
& Standard Dev. & 10 & 23 & 28 & 30 \\
\hline
\end{tabular}

\section{DISCUSSION}

Although the average change in the number of seeds, MUD and MRD shows consistent results, the individual dosimetry results vary considerably among patients. The results may be influenced by the prostate shapes and volumes chosen, as well as the random Gaussian distribution of seed displacement. We suggest implanting seeds from right to left periphery, and then from posterior to anterior. Although the seed positions in the periphery may be altered intraoperatively due to prostate edema and tissue movement, the inverse planning system can still optimize the seed positions in the center of the prostate to ensure that dose to the urethra and rectum is minimized as much as possible.

The results indicate that DITP may require more seeds and more needles than the preplan. In current clinical practice, approximately $10 \%$ extra seeds are ordered as a safety margin. Our study shows that the first three reoptimizations require about $5 \%(\mathrm{I}-125)$ and $11 \%(\mathrm{Pd}-103)$ extra seeds on average. However, it varies significantly among patients.

We obtained individual seed displacement from the simulation results of ten cases. The standard deviation in the $\mathrm{x}, \mathrm{y}, \mathrm{z}$ directions are $0.15 \mathrm{~cm}, 0.17 \mathrm{~cm}$, 
$0.26 \mathrm{~cm}$, respectively. This is the same as the results of seed reconstruction from matching planned seed positions to $\mathrm{CT}$ identified seed positions. In this study, the CT images were retrieved one day after the prostate brachytherapy in the OR. Edema of the prostate one day after brachytherapy may be more pronounced than that during brachytherapy or several days after brachytherapy [9]. DITP is a procedure in the OR so the standard deviation of seed displacement would be different from what we obtained in this study.

Intraoperative edema of the prostate is another factor that cannot be neglect in DITP. Many researchers are studying the effects of prostate edema on post-implant dosimetry. It was found that edema can result in a wide range of volume increases [10], and both the magnitude and duration of edema vary considerably among patients [11]. In this study we assume the prostate shape to be unchanged and only consider the effect of seed displacement on the re-optimization. In the future we will include prostate edema as observed in the OR at the re-optimization intervals.

The first step of needle optimization in the planning engine eliminates the needles that are near already implanted seeds. We found that the distance used to gauge needles will affect the optimization results. Because the template grid spacing is $5 \mathrm{~mm}$ and the standard deviation of seed displacement is $1.5 \mathrm{~mm}$ and $1.7 \mathrm{~mm}$ in $\mathrm{x}$, $\mathrm{y}$ direction, we chose $2.5 \mathrm{~mm}$ as the gauge. We also tested $1 \mathrm{~mm}$ and $4 \mathrm{~mm}$ and found that for the smaller prostate the $4 \mathrm{~mm}$ distance is too restrictive causing loss of some potential seed positions, and for the larger prostate the $1 \mathrm{~mm}$ may create too many potential seed positions so that the GA-based inverse planning engine would not produce optimal results in a limited number of generations (200).

Our simulated DITP divides the whole procedure into four reoptimization procedures. The demand for re-optimization depends on practical situations in the $\mathrm{OR}$, such as the observed edema and deformation of the prostate and the detected seed displacement. Our robotic system is designed to obtain real-time information including prostate imaging and implanted seed positions, and to update the dosimetry on-the-fly. Based on this information, the clinicians will decide if it is necessary to re-optimize the dosimetry. 


\section{CONCLUSION}

Simulated DITP based on a modified GA inverse planning engine generated increased average number of seeds, increased urethral dose and rectal dose during four re-optimization steps. Two types of seeds were tested and I-125 produced relatively lower average number of seeds, urethral dose and rectal dose than Pd-103. There was significant variability among the test cases.

\section{ACKNOWLEDGEMENT}

Work supported by NCI grant R01 CA091763.

\section{REFERENCES}

[1] Nag S, el al. Intraoperative planning and evaluation of permanent prostate brachytherapy: report of the American Brachytherapy Society. Int J Radiat Oncol Biol Phys. 2001, 51(5):1422 1430.

[2] Lee EK, el al. Intraoperative dynamic dose optimization in permanent prostate implants. Int J Radiat Oncol Biol Phys. 2003, 56(3):854 861.

[3] Todor DA, el al. Intraoperative dynamic dosimetry for prostate implants. Phys Med Biol. 2003, 48(9):1153 1171.

[4] Taschereau R, el al. Monte Carlo simulations of prostate implants to improve dosimetry and compare planning methods. Med Phys. 1999, 26(9):1952 1959.

[5] $\mathrm{Yu} \mathrm{Y,} \mathrm{el} \mathrm{al.} \mathrm{A} \mathrm{genetic} \mathrm{algorithm} \mathrm{for} \mathrm{the} \mathrm{optimization} \mathrm{of} \mathrm{prostate} \mathrm{implants.} \mathrm{Med} \mathrm{Phys.}$ 1996, 23(12):2085 2091.

[6] $\mathrm{Yu} \mathrm{Y}$, et al. Automated treatment planning engine for prostate seed implant brachytherapy. Int J Radiat Oncol Biol Phys. 1999, 43(3):647 652.

[7] Fu L, el al. Bouquet brachytherapy: feasibility and optimization of conically spaced implants. Brachytherapy. 2005, 4(1):59 63

[8] Messing EM, et al. Intraoperative optimized inverse planning for prostate brachytherapy: early experience. Int J Radiat Oncol Biol Phys. 1999, 44(4):801 808.

[9] Dogan N, el al. Effect of prostatic edema on CT-based postimplant dosimetry. Int J Radiat Oncol Biol Phys. 2002, 53(2):483 489.

[10] Yamada Y, el al. Impact of intraoperative edema during transperineal permanent prostate brachytherapy on computer-optimized and preimplant planning techniques. Am J Clin Oncol. 2003, 26(5):e130 - 135.

[11] Waterman F.M, et al. Edema associated with $125 \mathrm{I}$ or 103Pd prostate brachytherapy and its impact on post-implant dosimetry: An analysis based on serial CT acquisition. Int $\mathrm{J}$ Radiat Oncol Biol Phys 1998, 41(4):1069 1077. 